\title{
Dalle strutture al testo. Errori in Italiano L2 di apprendenti cechi e slovacchi: osservazioni preliminari in prospettiva longitudinale
}

\author{
From Structures to Text. Errors in Italian L2 by Czech and Slovak \\ Learners: A Preliminary Analysis with a Longitudinal Perspective
}

VAleria de Tommaso [detommaso@mail.muni.cz]

Masarykova univerzita, Repubblica Ceca

\section{RiassunTO}

Nell'ambito degli studi sull'apprendimento di una L2, l'analisi dell'errore (EA) dà un contributo fondamentale sia allo studio dell'interlingua sia all'osservazione del processo di acquisizione della lingua straniera. Tenendo conto sia dei recenti sviluppi dell'EA basata sui corpora sia delle ricerche specifiche per l'italiano L2, il presente studio si propone di analizzare, in prospettiva contrastiva, gli errori rilevati nelle produzioni scritte di due apprendenti cechi e due apprendenti slovacchi di italiano L2 durante il loro percorso di acquisizione da iniziale ad avanzato. I dati sono stati raccolti durante un periodo di tre anni. L’obiettivo della ricerca è duplice: da una parte si vuole leggere le devianze alla luce dei risultati emersi in linguistica acquisizionale per l'italiano L2; dall'altra, si intende osservare costanti e cambiamenti negli errori per acquisire ulteriori dati sulla competenza transitoria di apprendenti slavofoni.

\section{Parole Chiave}

Analisi dell'errore; testualità; italiano L2; lingue slave

\begin{abstract}
Error analysis (EA) helps to understand the learner's interlanguage and helps to shed light on the process of L2 acquisition. Taking into consideration the recent corpus-based researches in EA and considering also the specific studies in the language learning field, in this article I analyze errors in written texts by two Czech and two Slovak students of Italian L2, from elementary to advanced level. Data have been collected in a period of three years and they are investigated in a contrastive perspective. This analysis has two main purposes: firstly, to observe errors in light of second language acquisition studies of Italian L2; secondly, to focus on errors in the different levels of interlanguage in order to gain new data in the transitional competence of learners with Slavic L1s.
\end{abstract}

\section{KeYWORDS}

Error analysis; textuality; Italian L2; Slavic languages

RICEVUTO 2019-10-09; ACCETTATO 2020-01-25 


\section{Introduzione}

L'analisi dell'errore (EA) è stato uno dei primi strumenti utilizzati per indagare le produzioni linguistiche degli apprendenti ed è innegabile che esso dia il suo contributo allo studio dell'interlingua; inoltre, come in Ellis (2008: 61), «a study of how learner's error change from one stage to another can shed light on the process of L2 acquisition». A partire dagli anni Cinquanta del secolo scorso, le ricerche sull'EA hanno perseguito due sostanziali scopi (Pallotti 1998; Cattana \& Nesci 2004): con il primo, didattico, si è messa in evidenza la necessità di ricercare e analizzare l'errore per trovare risposte adeguate a prevenirlo e/o eliminarlo; il secondo scopo, teorico, considera l'errore come elemento proprio della lingua dell'apprendente e, più in generale, come elemento utile a seguire il processo di acquisizione di una lingua, sia essa L1 oppure L2 (Corder 1981). In particolare, con gli studi di Selinker, e con l'introduzione del concetto di interlingua ${ }^{1}$ (IL), si è sottolineato il valore creativo dell'errore, che risulta essere l'effetto del tentativo di riprodurre la norma della lingua target; esso è una parte integrante del sistema linguistico del parlante/apprendente. Oggi, analizzare l'errore - inteso come segnalatore di «distanza tra produzione reale e produzione ideale» (Pallotti 2005: snp) - significa «descrivere la natura dell'interlingua dello studente e confrontarla con la lingua d'arrivo» (Cattana \& Nesci 2004: 35).

\section{Lo studio}

Attualmente non risultano disponibili studi specifici sull'analisi dellerrore da parte di apprendenti cechi e slovacchi di italiano L2. ${ }^{2}$ Si possono tuttavia citare studi sull'errore e sull'apprendimento di spagnolo L2 (Rodríguez García 2014 e in stampa), ricerche di tipo contrastivo basate sulla descrizione di lingue tipologicamente diverse come il ceco, lo slovacco, l’inglese e l'italiano (Klimová 2011; Klímová 2011 e 2013) e sull'apprendimento della temporalità e aspettualità dei verbi per l'italiano L2 (Bitonti \& De Tommaso, in stampa), studi che mettono in evidenza le differenze tra alcuni aspetti dei sistemi linguistici delle lingue citate e le conseguenti ricadute che esse hanno sul piano acquisizionale e glottodidattico.

Procedendo in ottica contrastiva, il presente studio si propone di analizzare gli errori rilevati nelle produzioni scritte di quattro studenti universitari cechi e slovacchi di italiano L2 durante il loro percorso di acquisizione da elementare ad avanzato, percorso corrispondente ai primi tre anni di studio (laurea triennale). ${ }^{3}$ Lanalisi è di tipo qualitativo e non quantitativo ${ }^{4}$ : considerando le varietà dell'interlingua, si osserveranno le singole aree della lingua nelle quali si manifesta l'errore,

1 Selinker, L. (1972). Interlanguage. International Review of Applied Linguistics. 10, 209-31, citato in Pallotti (1998: 20).

2 Presso la Cattedra di Lingue Romanze dell'università di Olomouc è in corso di realizzazione un corpus di testi di studenti cechi e slovacchi disponibile online e a libero accesso (progetto Czech-It!, Linguistic corpus of native Czech learners acquiring Italian language, DOI 10.5281/ZENODO.824985). Lo scopo è quello di raccogliere materiale utile a studi di tipo contrastivo; non ci sono ancora analisi disponibili.

3 Questo studio è parte di una più ampia ricerca sullerrore, condotta da chi scrive, in testi scritti di apprendenti di italiano L2 con L1 ceca e slovacca, oggetto della tesi di dottorato dal titolo Analisi degli errori a livello sintattico in testi scritti da apprendenti cechi e slovacchi di italiano L2.

4 L'esiguo numero di informanti non permette di poter fornire generalizzazioni definitive. Il numero di testi analizzati, tuttavia, può considerarsi un punto di partenza per ricerche future (si veda, al riguardo, lo studio di Bozzone Costa 2002) . 
se ne seguiranno longitudinalmente i cambiamenti anche alla luce dei risultati riportati in linguistica acquisizionale; si rileveranno tipologie di errore specifiche e si ricercheranno nodi di criticità che siano utili a futuri approfondimenti e ricerche.

\subsection{La raccolta dei dati}

Per la raccolta dei dati sociolinguistici e dell'esecuzione del compito ci si è attenuti ai lavori di Dagneaux, Dennes \& Granger (1998), Bestgen, Granger \& Thewissen (2012) e Callies \& Götz (2015) i quali dimostrano come sia possibile superare i limiti attribuiti agli studi dell'EA con l'aiuto della linguistica dei corpora e con la creazione di banche di dati rigorose, costituite da subcorpora comparabili e interrogabili attraverso un sistema di tagging definito e applicato in modo univoco che permetta di superare la confusione a cui spesso la categorizzazione dell'errore è soggetta. ${ }^{5}$

I dati del corpus sono ricavati da 111 testi ottenuti somministrando 29 task nell'arco dei tre anni di studio della laurea triennale presso l'Università Masaryk di Brno: 8 testi sono stati prodotti in classe durante le ore di lezione e 34 testi in sede di esame, con restrizione di tempo e senza l'ausilio di dizionari cartacei o online; 69 testi sono stati scritti come esercitazioni da svolgere a casa, senza quindi restrizioni di tempo e con la libertà di consultare i materiali didattici. La scelta tematica dei task segue i contenuti dei programmi previsti per i vari corsi dei tre anni di studio. Si è deciso di somministrare task di forma testuale libera ${ }^{6}$ e che richiedano la scrittura di tipi testuali diversificati in linea con il livello linguistico degli apprendenti, così come definito dal Quadro Comune Europeo di Riferimento per le lingue (QCER 2002). Questo permette di delineare un quadro prestazionale sincronico per varietà di IL e di conseguenza fornire una base per una sintesi degli errori.

I task chiedevano la scrittura di diverse tipologie di testo; la differenziazione dei tipi di testo - in descrittivo, narrativo, espositivo, regolativo, argomentativo, e relativi sottogeneri - segue la descrizione proposta da Werlich ${ }^{7} .15$ testi sono stati prodotti durante il primo anno di corso (varierà IL elementare), 41 durante il primo semestre del secondo anno (IL intermedia iniziale), 19 durante il secondo semestre del secondo anno (IL intermedia) e 36 nel terzo anno (IL avanzata). ${ }^{8}$

Il riconoscimento e la classificazione degli errori ha portato alla creazione di un database di 1385 stringhe di testo la cui codificazione è stata elaborata all'interno del programma di analisi statistica IBM SPSS Statistics 25.

Per la raccolta dei dati sono state selezionate 4 informanti donne, due madrelingua ceche e due madrelingua slovacche, le quali hanno seguito un identico percorso di studi di lingua italiana tra gli a.a. 2015/16-2017/18 e che all'inizio del percorso di apprendimento erano principianti assolute.

5 Per una sintesi delle critiche imputate all'EA, si veda Ellis (2008: 60-61) e Andorno \& Rastelli (2009).

6 Cattana \& Nesci (2004: 54) puntualizzano che per poter ricostruire l'interlingua degli studenti in una certa fase del loro apprendimento è necessario analizzare gli errori su materiale prodotto per comunicare in modo libero, evitando cioè scelte imposte o guidate.

7 Ora anche in Ferrari (2014: 257-59), la quale puntualizza che «nella concretezza della produzione linguistica i tipi di testo, così come i generi, sono raramente omogenei».

8 L'indicazione delle varietà di IL segue le indicazioni del QCER (2002) e si basa sul confronto dei programmi dei corsi di lingua con i descrittori proposti nelle linee guida CILS (Barni \& al. 2009). 
Inizialmente è stato distribuito un questionario per la raccolta dei dati sociolinguistici dal quale emerge che tutte le quattro informanti hanno un'età compresa tra i 18 e i 20 anni e un'istruzione liceale conclusa con il massimo dei voti, non hanno genitori o parenti italiani, hanno un livello di conoscenza dichiarato dell'inglese uguale o superiore a B2. Altre lingue studiate sono il russo e il tedesco. Per quel che riguarda il contesto di apprendimento, le fonti dichiarano di studiare italiano solo presso l'Università Masaryk di Brno; esse pertanto utilizzano i medesimi testi di riferimento (una grammatica di tipo contrastivo ${ }^{9}$ e un corso di lingua di tipo comunicativo ${ }^{10}$ ) e hanno seguito le lezioni di lingua pratica delle medesime docenti (due madrelingua ceche, una madrelingua italiana). Le studentesse dichiarano inoltre che, fuori dalle aule universitarie, integrano lo studio della lingua con la lettura di testi con traduzione ceca/slovacca a fronte, ascoltano musica italiana, guardano film italiani sottotitolati e utilizzano applicazioni mobili.

I dati sociolinguistici, in conclusione, evidenziano un gruppo di informanti che, con la sola eccezione della L1 e L3, è qualitativamente omogeneo. ${ }^{11}$

\subsection{La classificazione degli errori}

Per una descrizione formale dellerrore il più possibile completa si è utilizzata la tassonomia della cosiddetta "strategia superficiale" integrata dall'indicazione delle categorie e degli elementi linguistici interessati (Cattana \& Nesci 2003: 77): si parlerà quindi di errori di omissione, aggiunta, scelta, ordine, malformazione; per le categorie linguistiche, si farà riferimento a errori di sintassi, lessico, ortografia, morfologia, morfologia/sintassi, punteggiatura. I casi dubbi - casi che richiedevano ricostruzioni plausibili multiple - non sono stati considerati. ${ }^{12}$

Nella categoria della sintassi sono stati inclusi errori di aggiunta/omissione/scelta di articoli e preposizioni (ess. 1-3); errori di accordo (es. 4); di concordanza dei tempi deittici e anaforici (es. 5); di aggiunta/omissione/scelta delle preposizioni con funzione connettivale (es. 6); di aggiunta di espressioni anaforiche referenziali non necessarie (es. 7); di aggiunta/omissione di pronomi (es. 8) e di scelta dei pronomi in riferimento al genere e numero del referente, alla sua funzione sintattica (es. 9) e alla forma forte/clitica; di scelta del modo del verbo nelle frasi subordinate (es. 10), dell'aspetto verbale (es. 11); di ordine degli elementi a livello sintagmatico (es.12) e di frase (es. 13).

(1) Mi trovo nell'aula molto grande, ci sono tante tavole

(2) Ma io ho aspettato altre tre giorni per ricevere i miei libri, che ho ricevuto con la molta gioia

(3) L'inno nazionale è stato scritto da Goffredo Mameli in 1847

(4) Buoni per il nostro digestione sono le lenticchie oppure diverse erbe

(5) E sono sorpresa che sono riuscita a dire alla gente cosa voglio, cosa non voglio e discutere e parlare in italiano

9 Bachniková, A., Benešová, H. \& Ehrenbergerová, L. (2008). Italština. Praha: Leda.

10 La Grassa, M. (2013). Italiano all'università 1. Roma: Edilingua.

11 Le due L1, in ogni caso, sono molto affini: per i punti di contatto e distanza a livello lessicale, sintattico, morfofonologico e pragmatico tra le due lingue, si veda Sokolová, Musilová \& Slančová (2005) e Trovesi (2013).

12 Per la categorizzazione degli errori, si è anche tenuto conto della proposta del progetto ICLE. Si veda Bestgen \& al. (2012: 113-15). 
(6) Il re ha cominciato di cercare un uomo felice

(7) Il padre di Natalia si chiama Miroslav, lui fa un designatore

(8) Nella mia stanza c’è uno scaffale di CD originali - tutte le sere, quando prepari la cena, ascolta[ne] almeno uno -

(9) Lo promette tutto che il giovane vuole ma poi il Re scopre che l'uomo contento non ha una camicia

(10) Non sono convinta che çè la soluzione per tutto nella vita degli uomini e delle donne in famiglia

(11) Non ho voluto ferirlo, ma non sono stata sicura con il passo così grande

(12) Io le ho dato un libro e al mio papa ho dato il nuovo frullatore

(13) Lo strudel cotto tagliate a fette, potete cospargerlo con zucchero a velo e servirlo tiepido

Per quel che riguarda gli errori lessicali, oltre alla scelta di una parola utilizzata al posto di unaltra (es. 14) e alle voci inventate o inesistenti (es. 15), questi comprendono anche la scelta di lessemi con funzione connettivale logica e testuale (congiunzioni e avverbi) (ess. 16-17) e gli errori di registro (es. 18).

(14) Mi piace rimanere fuori e paseggiare o leggere un libro, ma purtroppo non spendo molto tempo fuori se devo imparare per le lezioni

(15) e non cè niente male quando le persone seguono i suoi desideri e no le espetazioni della società

(16) Anche due settimane fa avevo soltanto 20 anni e non volevo sposarsi nessuno

(17) Il volo ha avuto di oltre dieci ore la tardazza grazie ad una seria d'inconvenienti tecnici

(18) Però voleva stare il vescovo. Niente!

Alla categoria dellortografia appartengono gli errori di errata grafia, come le mancate geminazioni (es.19), gli errori di malformazione, tra i quali l'unione/separazione di parole, l'omissione o l'aggiunta dell'apostrofo (es. 20) ${ }^{13}$ o il suo errato posizionamento, e la scelta delle varianti degli articoli determinativi e indeterminativi determinate dal suono iniziale delle parole che li seguono ${ }^{14}$ (es. 21).

(19) Allora penso che gli alimenti esenziali sono carne, patate, canederli e un po' di verdura

(20) Un fine settimana cuoce la moglie, un'altro il marito

(21) Anche per i adulti è comodo e non costa molto

Gli esempi (22-24) afferiscono rispettivamente a casi di morfologia, morfologia/sintassi e punteggiatura. Nella categoria della morfologia/sintassi sono stati inclusi gli errori che non è possibile attribuire con sicurezza alla morfologia o alla sintassi.

13 Si osservi che questo errore passerebbe del tutto inosservato in un testo orale.

14 La devianza viene considerata ortografica (e non sintattica) perché si ritiene che l'errore sia nella scelta della variante dell'articolo che dipende dal suo intorno fonologico (destro); di contro, a livello morfologico, l'apprendente ha riconosciuto correttamente i tratti sintattici di genere (maschile) e numero (plurale). 
(22) Quando mangievamo, sentivamo la musica e chiacchieravamo

(23) Si prepari per le riunione

(24) Honza ha 37 anni [,] cè designer come Adéla

L'esempio (22) è un caso di malformazione del verbo; la frase (23) mostra un tipo di deviazione che può essere interpretabile come sintattica (mancato accordo tra articolo e nome) o morfologica (formazione errata del plurale). Con (24) ci si trova davanti a un caso di omissione di un segno interpuntivo.

\section{Lanalisi dei dati: una prospettiva longitudinale degli errori}

Il Grafico 1 mostra i dati relativi alle frequenze delle singole aree della lingua nelle quali si manifesta l'errore nei quattro livelli di interlingua individuati: elementare, intermedio iniziale, intermedio e avanzato.

Il grafico mostra come durante l'intero percorso di studi la sintassi e il lessico costituiscano costantemente le principali aree di errore: insieme, queste aree comprendono il 59.8\% degli errori per IL elementare, il 73\% per IL intermedia iniziale, il $66.1 \%$ per IL intermedia e il $79.9 \%$ per IL avanzata. Il dato è - nonostante la leggera flessione nella fase intermedia - in costante crescita, a prova del fatto che con il progredire delle competenze testuali aumentano anche le possibilità di errore legate alle differenti dimensioni del testo (enunciativa, referenziale e logica, come in Ferrari 2014), dimensioni che coinvolgono soprattutto la categoria sintattica. ${ }^{15}$

Risultano marginali gli errori relativi alla morfologia/sintassi (tra l'1.4\% e il 3.5\%) che pertanto in questa sede non verranno presi in considerazione.

Un discorso a sé meritano le categorie di ortografia, morfologia e punteggiatura che, come risulta dal Grafico 1, sono posizionate nella fascia centrale e mostrano maggiore mobilità nella scala delle frequenze dell'errore con il procedere dell'apprendimento. Nella varietà elementare l'area dell'ortografia (16\%) presenta più errori di quella della morfologia (13.9\%) e della punteggiatura (8.3\%); nella varietà intermedia iniziale è la punteggiatura (11.5\%) a risultare meno corretta, seguita da ortografia (7.4\%) e morfologia (6.6\%); nella varietà intermedia è di nuovo lortografia $(15.7 \%)$, seguita da punteggiatura $(8.3 \%)$ e morfologia $(6.3 \%)$; infine nella IL più avanzata la morfologia $(6.8 \%)$ risulta dare maggiori difficoltà invece di, nellordine, punteggiatura $(6.2 \%)$ e ortografia (5.8\%).

È difficile in questa sede procedere a un'osservazione approfondita e sistematica degli errori per tutte le categorie lessicali e per tutte le varietà di IL; inoltre, per avere dati scientificamente più validi si dovrebbe operare su un numero maggiore di apprendenti. Si ricorda pertanto che l'analisi che

15 Questo dato conferma anche quanto in Chini (2005: 104) secondo la quale «l'evoluzione della competenza testuale è strettamente connessa a quella morfologica e sintattica». Osservando infatti «in positivo» le realizzazioni dell'errore (Pallotti 2005: snp), è evidente come le apprendenti inferiscano maggiormente ipotesi nell'applicazione delle loro conoscenze relativamente al livello sintattico. A titolo esemplificativo, si possono prendere in considerazione gli errori rilevati per i connettivi coordinanti e subordinanti: questa categoria è sporadicamente presente nella IL elementare - le cui competenze testuali sono ancora minime - e risulta più problematica a partire dalla IL intermedia iniziale, il cui livello rappresenta proprio lo stadio in cui si inizia a sviluppare in modo più sistematico la subordinazione (si vedano, al riguardo, i descrittori per i livelli A2 e B1 indicati per le certificazioni CILS in Barni \& al. 2009: 26-29). 


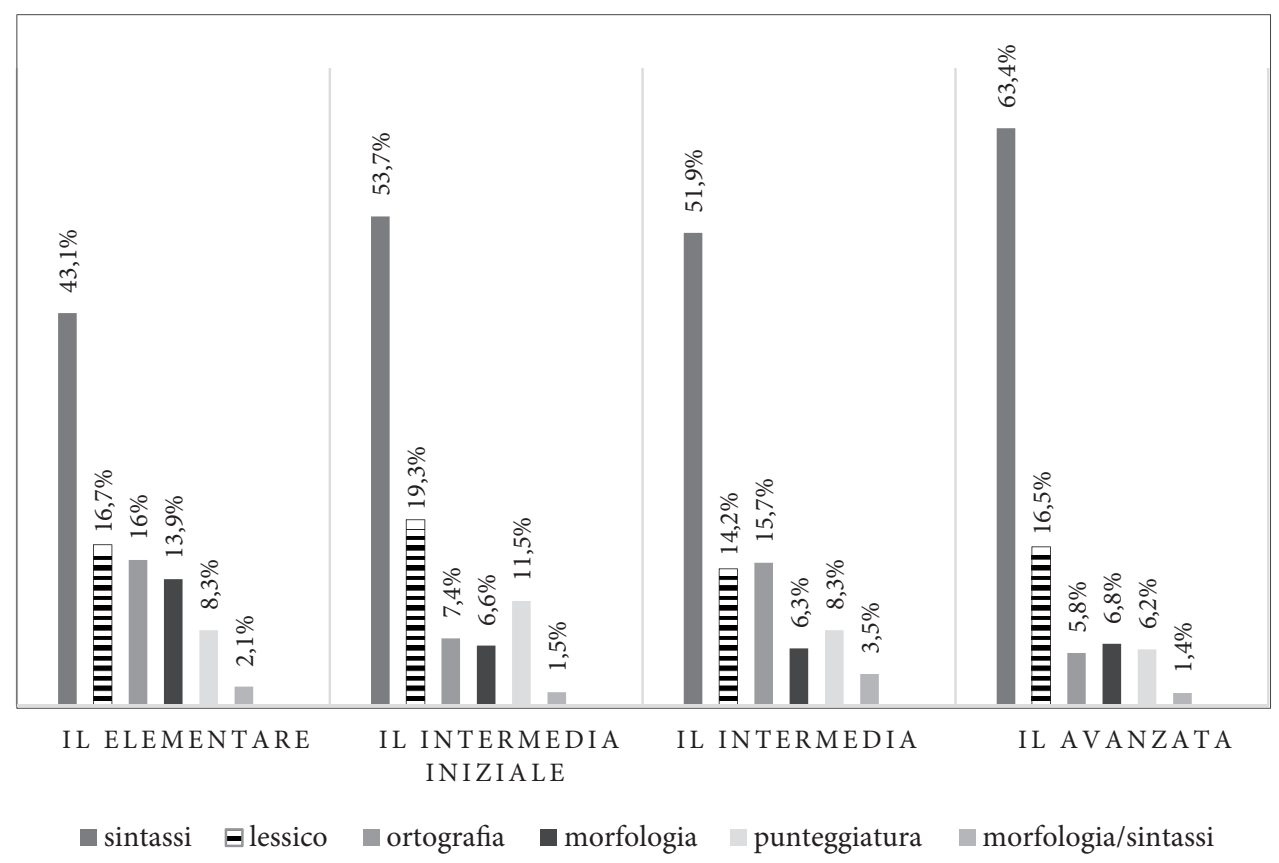

Grafico 1: Errori per area della lingua su base longitudinale

segue vuole essere qualitativa, con lo scopo di fornire indicazioni sulle aree di maggiore criticità per le due L1 nelle varie fasi dell'apprendimento e tentare un'interpretazione delle strategie messe in atto nella comunicazione scritta.

\subsection{Sintassi}

Gli errori di sintassi hanno una percentuale minore a livello elementare (43.1\%), fatto spiegabile con la brevità delle frasi, abbinata alla paratassi, propria delle produzioni scritte in questa fase dell'apprendimento. Essi aumentano con l'avvicinarsi della IL alla lingua target (53.7\% per IL intermedia iniziale, $51.9 \%$ per IL intermedia e $63.4 \%$ per IL avanzata).

Nella varietà elementare si osservano in maggior numero gli errori di scelta, aggiunta e omissione di articoli (soprattutto determinativi) e preposizioni. Si tratta di categorie linguistiche il cui rapporto forma/funzione nel ceco e nello slovacco, come in altre lingue slave, non corrisponde a quello dell'italiano: di fatto queste lingue non contemplano l'uso dell'articolo, riservando le indicazioni di determinatezza/indeterminatezza e referente noto/non noto a meccanismi linguistici di ordine pragmatico (Young 1999). Se si osservano le preposizioni, si assiste in particolar modo alla scelta errata con sovrauso di $d i$ e $a$, in linea con Calleri (2008), o all'omissione nei casi in cui fungono da connettivi subordinanti (per es., spesso gioca [ai ]gli giochi di computer; ho pensato 
della mia stagione preferita; abbiamo deciso [di]sederci fuori ${ }^{16}$. Per quel che riguarda l'accordo del genere e del numero, le idiosincrasie si manifestano non tanto a livello sintagmatico quanto di frase, tra soggetto e aggettivo predicativo. Si nota inoltre il mancato accordo tra aggettivo possessivo e referente di terza persona singolare o plurale: si tratta di un'evidente interferenza negativa da L1 che porta gli apprendenti a estendere la forma morfologica di terza persona singolare (suo, sua ecc.) ai casi in cui il possessore, corrispondente al soggetto della frase, è plurale (e richiederebbe la forma invariata loro $)^{17}$ come nellesempio seguente (26), che avrebbe dovuto essere 'Le madri inoltre escono con i loro figli e giocano'

(26) Le madre anche escono con i suoi figli e giocano

Questa idiosincrasia si manifesta contemporaneamente all'acquisizione dell'uso degli aggettivi possessivi per esprimere le relazioni di parentela, per poi tornare sporadicamente, come si vedrà, anche nei livelli successivi. La forza del transfer negativo emerge anche nell'esempio (27) in cui alla parola stagione, correttamente formata al femminile e accordata allarticolo e al pronome nella proposizione successiva, corrisponde in L1 un lemma maschile, che evidentemente esercita morfologicamente attrazione sull'aggettivo predicativo finale di entrambe le proposizioni.

(27) Quando imagino l’anno e le stagioni, non sono sicura qual è il mio preferito. Le amo tutti!

Un'altra classe lessicale che presenta incertezze nella IL elementare è quella dei pronomi, e in particolare nella forma forte che viene preferita a quella clitica (per es., Dopo la cena Michele a chiesto a me se voglio sposarlo) e nell'espressione del soggetto anche quando non necessario ${ }^{18}$ (si veda es. (7)). Minime, infine, in questa varietà, sono le devianze relative all'ordine dei costituenti (di aggettivi a funzione predicativa che vengono anteposti al nome) e alla scelta dell'aspetto verbale (con estensione del passato prossimo sull'imperfetto nella frase principale, come nell'esempio (12)) o del tempo verbale (con il mancato rispetto del rapporto di contemporaneità nel passato ed estensione del presente sull'imperfetto in frase subordinata, come nell'esempio (5)). ${ }^{19}$

Gli errori di aggiunta o omissione dell'articolo continuano a essere preponderanti anche nei livelli successivi e riguardano in particolar modo l'articolo determinativo. ${ }^{20}$ Nel livello intermedio iniziale si riscontrano aggiunte di determinativo con possessivi usati con nomi di relazione fami-

16 Ma l'influenza dell'inglese L2 per i primi due casi (in cui il verbo 'play' richiede un oggetto diretto e il verbo 'think' è seguito da preposizione) e della L1 nel secondo (nel ceco e nello slovacco le subordinate oggettive non sono introdotte da preposizione) è plausibile. Si veda anche infra per quel che concerne le IL successive.

17 In questa fase iniziale gli apprendenti potrebbero anche essere guidati da vicinanza orto-fonologica tra la forma in L1 'svůj' e la italiana suo.

18 In un caso come pronome soggetto compare anche la forma clitica lo: si potrebbe ipotizzare un calco da L1 in cui nel medesimo ruolo si trova il pronome 'to', che ha funzione di incapsulatore anaforico.

19 Anche per queste strutture è forte l'interferenza negativa della L1: in queste lingue nel sintagma nominale l'aggettivo ha posizione standard prima del nome (Uhlírová 2017) e l’espressione dell’aspetto verbale e della concordanza dei tempi è affidata a costruzioni basate sulla distinzione tra perfettivo/imperfettivo e sulla salienza del tempo verbale della frase reggente (cfr. Bitonti \& De Tommaso: in stampa).

20 Solo nel livello avanzato risulta omesso anche un numero non trascurabile di articoli indeterminativi (9 su 63 totali). 
liare, a indicare la non completa acquisizione della regola che, evidentemente, si manifesta con sovrauso del determinativo. Quest'ultimo, inoltre, viene sovraesteso al posto dell'indeterminativo in quasi tutti i casi di scelta: le informanti al proposito sembrano mostrare, ancora nel livello avanzato, una particolare incertezza nell'introdurre un elemento non noto o indeterminato lì dove il referente è accompagnato da un modificatore più o meno complesso postposto alla testa, come nell'esempio (28).

(28) Dopo questo enorme successo comincia l'era molto produttiva nella quale gira un film ogni anno

Come gli articoli, anche le preposizioni costituiscono una categoria costantemente oggetto di errore: si tratta soprattutto delle preposizioni in posizione valenziale che nei livelli intermedi (iniziale e non) sembrano subire un forte influsso della L1 (per es., vuole bene [al] il mio pianoforte;

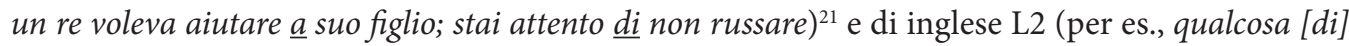
brutto; entra [in] una vigna; non dare niente a mangiare al mio gatto). Nel livello avanzato si assiste ancora a sovrauso e sovraestensione della preposizione di: l'aggiunta e la scelta di questa preposizione sembra dettata dalla funzione che le viene attribuita: quella specificativa e/o agentiva (per

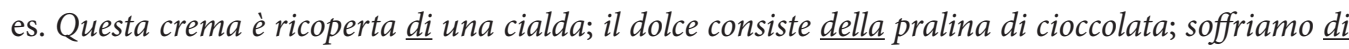
fame). Anche qui non mancano dei sospetti calchi da inglese L2 (per es., ho dovuto cercare per una camera libera), così da essere portati a concludere che l'espressione degli elementi di questa categoria grammaticale, ciascuno con vario peso semantico e grammaticale, sia fortemente condizionata da strategie di comunicazione interlinguistiche ${ }^{22}$ in tutte le fasi dell'apprendimento, anche nella più avanzata.

Per quel che riguarda le altre categorizzazioni nelle quali si è analizzata l'area della sintassi, l'esiguo numero dei dati non permette generalizzazioni; se ne dà tuttavia una sintetica rassegna:

- Lordine degli elementi nel passaggio verso la IL medio-avanzata acquista un peso sempre maggiore, a livello sintagmatico e di frase. Tra questi ultimi, si rilevano idiosincrasie nella gestione dell'informazione tra tema/rema e dato/nuovo, con relativa mancanza del clitico di ripresa (per es., Il semestre passato ho trascorso a Bristol; Il burro poi aggiungiamo ai biscotti e mischiamo con una mestola).

- Lerrata posizione dell'aggettivo rispetto al nome (attributivo o predicativo, per es. Carlo Verdone è un unico personaggio della commedia italiana) è presente fino alla varietà avanzata.

- La posizione degli avverbi interni ai sintagmi (per es., Ma non ci sempre riesco; Solo penso che...; Faccio attenzione alla mia dieta alimentare per rimanere in linea di più) non rispecchia quella della lingua target ancora nella IL intermedia.

- La posizione del clitico si lontano dal suo verbo (per es., Ma è necessario di dire che questa idea si gradualmente cambi) è un evidente segnale di transfer negativo da L1.

- Gli errori di accordo di numero tra aggettivo possessivo e referente di terza persona quasi

21 Nelle L1 i tre verbi richiedono, rispettivamente, un oggetto diretto, un oggetto indiretto e una proposizione finale introdotta da connettivo.

22 Al riguardo Callerri (2008: 49) già notava che per gli apprendenti di italiano L2 il compito di «riconfigurare rapporti tra elementi [...] può essere agevolato da fattori quali l'alta incidenza di un elemento nell'input ma anche, naturalmente, la paragonabilità delle strutture (il peso della L1 risulta proprio qui, evidentemente, determinante)». 
scompaiono nelle IL medio-avanzate. Persistono invece i mancati accordi nel numero e nel genere tra participio passato del verbo e referente soggetto.

- Laccordo tra soggetto e verbo non si realizza nei casi in cui il soggetto presenta espansione (per es., Carlo Verdone [...] insieme al fratello minore Luca si avvicinano al mondo del cinema): l'effetto del transfer negativo qui è evidente poiché la sintassi ceca ammette doppia possibilità di accordo.

- Laggiunta o l'omissione del pronome locativo nei casi che richiedono, rispettivamente, l'uso del verbo essere con valore copulativo o presentativo (per es., Questo model della vita çè sempre qui: nella mia mente, nel mio paese) si notano sporadicamente fin nel livello avanzato.

- Laccordo di genere e numero in presenza di costruzioni impersonali (per es., Però si deve stare attento a non ommettere niente di importante) è proprio della IL più avanzata.

Le deviazioni legate all'espressione dell'aspetto verbale e alla scelta dei tempi per esprimere i rapporti temporali in frasi complesse sono riscontrate in tutte le IL a partire dalla intermedia iniziale. Seppur non rappresentate in questo corpus da un numero significativo di occorrenze, esse evidenziano la difficoltà dell'apprendimento di queste categorie e l'interferenza del sistema della L1 (si veda anche Bitonti \& De Tommaso: in stampa).

\subsection{Lessico}

Relativamente al lessico, le devianze rintracciate si riferiscono sostanzialmente a errori di scelta del lemma. Inizialmente, come anticipato in 2.2, si è tenuta distinta la classe delle espressioni connettivali (perché più legate alla dimensione logica e sintattica del testo) e dello stile e registro (perché afferenti piuttosto alla dimensione pragmatica e testuale).

Gli errori di registro risultano assenti nella IL elementare, presenti nella IL intermedia iniziale (11 su 81 devianze totali) ${ }^{23}$ e minime (non oltre le 2 occorrenze) nella IL medio-avanzata.

Le scelte legate alle espressioni connettivali riguardano in generale sia congiunzioni (quando, mentre, perché, ecc. Per es., Quando mangievamo, sentivamo la musica e chiacchieravamo) sia avverbi (inizialmente, infine, ecc. Per es., Finalmente il uomo felice, che ha trovato, non aveva la camicia); si contano anche avverbi con funzione focalizzante come anche, proprio, già ecc. (per es., Nella Slovacchia c’è anche necessario, anche di questi tempi, di mostrare alla gente che gli uomini non devono sempre fare solo le attività tipicamente considerate maschili).

Nella IL elementare iniziano a manifestarsi le incertezze nella scelta di già e ancora (e il cui uso è legato alla polarità della frase. Per es., I nonni sono ancora in pensione) e l'uso sovraesteso legato ad approssimazione semantica ${ }^{24}$ di quando per mentre e di anche per inoltre (es. 29).

23 A determinare la forte presenza di queste espressioni non target nel livello intermedio iniziale è stata evidentemente la scrittura di due testi espositivi che partivano rispettivamente dalla lettura di una fiaba e dall'ascolto e analisi di una canzone (Ho visto un re di Dario Fo e Enzo Jannacci) che conteneva anche alcune espressioni dialettali milanesi. Nella rielaborazione del testo nessuna delle scriventi ha tenuto conto del fatto che il genere testuale richiesto - di tipo espositivo - non ammette reduplicazioni espressive (cercava e cercava) e parole diatopicamente marcate ('villano' per contadino, 'sciur' per signore), rimanendo così legate ad espressioni proprie del testo di partenza.

24 In questa sezione si farà riferimento a errori legati a uso di genericismi, estensione e approssimazione semantica, e perifrasi analitiche. I genericismi si ritrovano nell'uso di verbi con bassa specificità semantica come essere, avere, fare che 
(29) Lui era molto elegante, anche è andato nella sua nuova macchina e mi ha preso in un ristorante caro.

Nel passaggio alla varietà intermedia, sviluppandosi la subordinazione, si aggiunge la sovraestensione di perché su altri connettivi logici come perciò, infatti, quindi, per questo e di quando su che (per es., Poi ci abbiamo dati i presenti, ma adesso, quando siamo tutti adulti, i presenti sono solo piccoli.). Nelle varietà medio-avanzate la subordinazione risulta decisamente più ricca e più diversificate sono anche le estensioni: se da una parte il confine semantico di perché risulta stabilizzato (è presente un solo caso, scambiato con siccome), dall'altra si assiste a frequenti scambi tra congiunzioni che hanno tratto semico opposto (grazie a invece di a causa di, tranne invece di oltre a) o che condividono solo alcuni tratti semantici (dopo invece di da quando, fino a invece di ancora. Per es., Ma dopo questa dieta è finita sono più o meno in buona salute, allora mangio normalmente). In questa fase l'interferenza interna al sistema determina anche scambio di elementi che costituiscono coppie lessicali formalmente o semanticamente simili come particolarmente/in particolar modo, in maggioranza/soprattutto, alla fine/finalmente, brevemente/ in breve (per es., Le moglie si usualmente curano soprattutto dei bambini e di tutto che ci entra e anche delle pulizie, del bucato e della cottura. Brevemente tutti lavori domestici).

Per quel che riguarda infine la scelta di nomi, aggettivi e verbi, in linea con i risultati di Bozzone Costa $(2002)^{25}$, nella varietà elementare e intermedia iniziale si assiste a deviazioni prodotte da strategie semplificative diversificate intralinguistiche e al ricorso a calchi e prestiti da L1 o inglese L2. Questi ultimi non sono frequenti (a titolo esemplificativo si veda nuovamente l'esempio (29), prendere $[q c]$ in un ristorante invece di portare $[q c]$ in un ristorante; fare le gite nella natura invece di fare le gite in campagna/fuori città; avere la cena invece di cenare ${ }^{26}$; spendere il tempo invece di passare il tempo) e sono quasi assenti nelle varietà medio-avanzate, considerando anche il fatto che il testo scritto permette rielaborazione del messaggio e che, in molti casi, si aveva libero accesso a dizionari.

Rimanendo nell'ambito delle strategie intralinguistiche, nella varierà elementare e intermedia iniziale si riscontrano genericismi (la sovraestensione di verbi delessicali -soprattutto avere - per es. ho il compleanno ${ }^{27}$ ), estensioni che riguardano coppie sinonimiche (imparare per studiare, passare e andare per arrivare, viaggiare per andare, credere per avere fiducia, anche con relazione associativa come straniero per estraneo, scuola per universita ${ }^{28}$ ) e perifrasi analitiche (giochi di

\footnotetext{
vengono sovraestesi e scambiati tra loro «conferendo al testo scritto carattere di imprecisione e di forte lontananza dalla lingua target»; gli errori di estensione/approssimazione semantica riguardano «la scelta di una parola che appartiene alla stessa area semantica dell'item di L2 e che ne condivide alcuni tratti semici, realizzando relazioni di contiguità, di iponimia e di sinonimia che violano alcune regole collocazionali e di restrizione di selezione», cosa che accade per esempio con le coppie di verbi venire/andare, sapere/conoscere ecc.; gli errori legati alle perifrasi analitiche sono dovuti a «lacune lessicali» e sono preferite a «entrate lessicali indipendenti [...] anche come strategie pre-morfologiche in sostituzione di parole codificate in italiano con morfologia» (Bozzone Costa 2002: 42-49).

25 Si tratta di uno studio longitudinale degli errori lessicali raccolti da 120 produzioni scritte prodotte da apprendenti adulti di diverse L1 e suddivisi per varietà di apprendimento (elementare, intermedia, avanzata).

26 Anche questo esempio mostra che non è sempre possibile stabilire in modo univoco il ricorso a una strategia: in questo caso potrebbe trattarsi di uso sovraesteso del delessicale avere.

27 Il dubbio però che sia un calco da L1 è legittimo perché corrispondente all'espressione corrente 'mám narozeniny'.

28 Anche per questo esempio l'ambiguità è forte: potrebbe trattarsi con molta probabilità anche di calco da L1, proprio perché la parola 'škola' ha tra i suoi referenti 'università.
} 
computer per giochi elettronici, avere un pasto buonissimo per cenare benissimo). Si menzionano anche i tentativi mal riusciti guidati da assonanze (affitti invece di soffitti) o similarità (bronzare invece di abbronzarsi).

Con il procedere dell'acquisizione nella fase intermedia si riducono decisamente i genericismi, che tornano però nella fase avanzata ${ }^{29}$ (per es., Le scrivo per chiedere un risarcimento per la mia male sperienza con la vostra compania aerea, 'negativa') e persistono le devianze dovute ad approssimazione semantica per parole appartenenti alla stessa famiglia lessicale (cuocere per cucinare, cibo per piatto, assicurare per sostenere, serata per sera, allenatore per maestro). Sempre all'interno delle famiglie lessicali si assiste anche ad attribuzione errata del suffisso morfologico in aggettivi, avverbi e nomi derivati (inaspettabile per inaspettato, cantatore per cantante, intensivo per intenso, terrificantemente per terrificante).

\subsection{Ortografia}

La terza area della lingua che mostra punti di criticità è l'ortografia. Emergono soprattutto deviazioni nella scrittura degli accenti, delle geminate e degli articoli che dipendono dal suono iniziale delle parole che li seguono.

Nel livello elementare e intermedio iniziale (16\% e 7.4\% del totale) ci sono più incertezze nella resa delle consonati geminate (paseggiare invece di passeggiare), in secondo luogo nelle varianti degli articoli indeterminativi maschili (un studente invece di uno studente), in terzo luogo nella scrittura degli accenti (dittà invece di ditta). Nella IL intermedia iniziale, oltre alla scrittura delle consonanti geminate risulta meno corretta la resa delle desinenze in -chi e -ghi (si dedici invece di si dedichi). Differenti sono anche gli errori legati alla malformazione, perché più legati al fenomeno dell'elisione: più problematiche infatti risultano le varianti che richiedono o non richiedono l'apostrofo (un'po invece di un po'). Marginale, infine, è la presenza della scelta della maiuscola in luogo della minuscola.

Anche nella IL intermedia ( $15.7 \%$ del totale) si rileva la presenza dello stesso ordine di categorie e, ancora in modo preponderante, emergono gli errori di ortografia legati alla scrittura delle geminate. Persistono inoltre sia le incertezze tra le varianti morfologiche ( $i$ invece di $g l i$ ), sia le incertezze nella resa dell'elisione (un'uomo invece di un uomo).

Infine, la IL avanzata (5.8\%) rivela ancora errori nella scrittura delle geminate (20 occorrenze su 30 totali); quasi superati, ormai, risultano gli errori di malformazione e accento.

Analizzando questi dati longitudinalmente si può osservare che la scrittura delle consonanti geminate si rivela instabile e persiste anche nella fase più avanzata dell'apprendimento seppure con frequenza sensibilmente minore. Molto probabilmente queste idiosincrasie derivano dal fatto che nelle lingue ceca e slovacca la durata delle consonanti non ha valore distintivo e la geminazione è prevalentemente legata a cause di morfologia derivazionale (aggettivi denominali e gradi di comparazione).$^{30} \mathrm{Si}$ esauriscono, invece, con il progredire delle conoscenze e il consolidarsi della

29 Si potrebbe ipotizzare per questo livello di IL anche un minore controllo del monitor o una tendenza a ricorrere a espressioni proprie del parlato colloquiale.

$30 \quad$ Pravidla českého pravopisu (1994: 36-37). 
morfologia, le instabilità attribuite ai fenomeni dell'elisione e alla scelta delle varianti morfologiche degli articoli determinativi e indeterminativi.

\subsection{Morfologia}

Anche per la categoria della morfologia è possibile osservare gli elementi devianti. Essi coprono il $13.9 \%$ degli errori nella varietà elementare, il 6.6\% in quella intermedia iniziale, il $6.3 \%$ e il 6.8\% nelle varietà intermedia e avanzata. Da questi numeri è evidente quindi che le devianze più numerose si presentano nel livello elementare.

In questa categoria è il verbo l'elemento più problematico durante il percorso di apprendimento. Nello stadio iniziale questo si evidenzia per la coniugazione del presente indicativo (lui esca invece di lui esce) e imperfetto (mangievamo invece di mangiavamo) e nella resa del participio passato dei verbi (ho interrompeto invece di ho interrotto; ho metto invece di ho messo). Nel livello intermedio iniziale le informanti sbagliano le forme dell'imperativo (non $f a$ ' invece di non fare; lavi $i$ piatti invece di lava i piatti) e mostrano ancora incertezze per il tempo presente indicativo (sceglio invece di scelgo). Si evidenziano, in questo secondo gruppo di testi, errori che coinvolgono i verbi copulativi e quelli intransitivi: vengono infatti aggiunti, probabilmente per influenza della L1, i pronomi personali atoni (si diventa, come nel ceco 'stává se', invece di diventa; si cambia, in ceco 'mění se', invece di cambia; i casi contenuti nel corpus riguardano la $3^{\text {a }}$ persona singolare e plurale). Questi errori probabilmente sono da attribuirsi a un vocabolario non ancora definitivamente acquisito, indipendentemente dalla frequenza dell'input. ${ }^{31} \mathrm{Ma}$ la tendenza continua nelle varietà medio-avanzate, in cui la quasi totalità degli errori morfologici sui verbi si concentra proprio nell'aggiunta del pronome personale atono, e questo anche per verbi di alto uso, appresi già in fase iniziale.

Per riassumere, in linea con le sequenze acquisizionali relative all'espressione della temporalità $\mathrm{e}$ della modalità, ${ }^{32}$ è evidente come, nelle fasi iniziali, si stiano ancora consolidando sia i primi tempi verbali (presente, passato prossimo, imperfetto) sia la modalità deontica. Gli errori nei livelli più avanzati, relativi all'aggiunta del pronome personale atono a verbi copulativi e intransitivi che non lo richiedono, sono invece presumibilmente dovuti a transfer da L1 o a fossilizzazione.

Per quel che riguarda gli errori nel genere di nomi e aggettivi, le frequenze sono minime; eppure è possibile tracciare delle linee di tendenza: per tutti e quattro i livelli si tratta sostanzialmente di errata attribuzione di genere dovuta probabilmente a transfer negativo (la bagna invece di il bagno, i vitamini invece di le vitamine) o, nei casi più avanzati, si potrebbe ipotizzare anche un minore controllo del monitor, specialmente per quelle parole che sono di alto uso e che sono già introdotte nei livelli iniziali. Si osservi l'esempio 30:

31 È utile qui aggiungere che nei risultati relativi agli studi della sequenza acquisizionale dei pronomi atoni, il pronome personale $s i$ con funzione passivante, impersonale e riflessiva, è tra i primi ad essere appreso, dopo il ci presentativo e il $m i$ dativo. (Cfr. Berretta, M. (1986). Per uno studio dell'apprendimento dell'italiano in contesto naturale: il caso dei pronomi personali atoni. In A. Giacalone Ramat (Ed.), Lapprendimento spontaneo di una seconda lingua, Bologna: Il Mulino, citato in Chini 2005: 95.)

32 Per una sintesi delle sequenze acquisizionali dellespressione della temporalità e della modalità, si veda Chini (2005: 96-99). 
(30) Molte persone temono anche che se smettono di mangiare $\underline{\text { il carne, }}$ gli mancheranno alcune vitamine

La parola vitamine - a cui le altre informanti hanno attribuito il genere maschile - qui presenta una corretta espressione del genere; non così per la parola il carne, che potrebbe ritenersi transfer o lapsus dovuto a mancanza di attenzione, che evidentemente si è concentrata nella parte terminale della frase. Gli errori di numero risultano sensibilmente ridotti rispetto a quelli di genere, confermando quanto in Giacalone Ramat (2003: 25 e 37-51) sulle sequenze acquisizionali del nome in riferimento agli universali tipologici, secondo cui il numero è categoria meno marcata del genere e per questo pone meno difficoltà nell'apprendimento. Gli errori nell'espressione del numero $^{33}$ riguardano soprattutto la desinenza - e usata al posto di - $i$ ( $i$ pesce, le moglie invece di $i$ pesci, le mogli) e si può pensare che possano essere indotti da aggettivi e/o articoli in -e limitrofi: le grande macchine. Anche le incertezze sulle desinenze - e e - $i$ del plurale confermano le sequenze acquisizionali. Minime risultano le occorrenze degli errori di scelta dell'ausiliare o dell'espressione del grado dell'aggettivo (meglio invece di migliore).

\subsection{Punteggiatura}

In ultima analisi si è presa in considerazione la categoria della punteggiatura. Dal Grafico 1 è evidente che gli errori di punteggiatura si concentrano nella IL intermedia iniziale: infatti, è in questa fase dell'apprendimento che la subordinazione inizia a farsi più presente. ${ }^{34}$ Le devianze corrispondono all'11.5\% del totale e riguardano in particolare la gestione della virgola davanti a subordinate (aggiunta) e in apposizioni (omissione). Qui è anche evidente il transfer della L1 nell'uso interpuntorio: le subordinate, in ceco e slovacco, richiedono obbligatoriamente l'uso della virgola davanti al connettivo subordinante. Nello specifico, i problemi maggiori si evidenziano con le subordinate completive e relative (es. 31).

(31) Ma devo confessare ${ }_{2}$ che l'estate è meglio di tutti

(32) La prima cosa che è venuta in mia mente quando ho pensato della mia stagione preferita è stata "l'estate", ma quando faccio un raggionamento, creedo che non è l'estate, è la primavera

(33) Inoltre, il cibo carne può anche contenere contaminanti come ormoni, erbicidi e pesticidi e antibiotici, i mangiatori di carne devono anche affrontare i rischi di virus, batteri e parassiti come la salmonella, la trichinella e altri vermi e parassiti della toxoplasmosi.

In generale, sin dal primo livello, si può osservare un mancato riconoscimento delle funzioni sintattiche e logiche dei segni interpuntivi ${ }^{35}$ : nelle varietà elementare e intermedia iniziale le informanti abusano soprattutto della virgola nei casi che richiederebbero i due punti o segni interpuntivi più forti (punto e virgola, o punto) (es. 32); a partire dalla IL intermedia iniziale, quando

33 Per una sintesi delle sequenze acquisizionali dell'espressione del numero, si veda Chini (2005: 100).

34 In questa fase, in modo produttivo gli apprendenti sviluppano le frasi complesse come relative e completive, subordinate causali, temporali, dichiarative, ipotetiche introdotte da se (Barni \& al. 2009: 26-29)

Per le funzioni della punteggiatura si veda Ferrari \& Zampese (2016: 295-311). 
il periodare inizia a farsi più lungo e complesso, le informanti mostrano di non saper realizzare le funzioni informative della punteggiatura: si evidenziano apposizioni e incisi malformati e l'impaccio a gestire elenchi complessi e argomentazioni, anche in fase avanzata (es. 33).

\section{Osservazioni finali}

Lanalisi degli errori in testi scritti prodotti da due informanti ceche e da due informanti slovacche di italiano L2 durante il loro percorso di apprendimento da elementare ad avanzato, in parte ha confermato i risultati già illustrati negli studi di linguistica acquisizionale, in parte ha evidenziato aree di criticità che meritano ancora interesse.

Dalle riflessioni sulle strategie messe in atto nella comunicazione scritta sono emerse numerose interferenze negative da L1 e da inglese $\mathrm{L}^{36}{ }^{36}$, nei casi in cui il transfer non è evidente, il dubbio risulta legittimo.

Losservazione longitudinale dei dati ha portato a rilevare come, in tutti i gruppi di testi, gli errori di sintassi e lessico costituiscano più del $50 \%$ del totale, in accordo con Cattana \& Nesci (2004). Le aree che longitudinalmente hanno manifestato più variazione di frequenza tra i livelli di IL sono state quelle dellortografia, della morfologia e della punteggiatura.

I dati sulla sintassi hanno messo in evidenza usi idiosincratici dellarticolo, delle preposizioni e dei pronomi, deviazioni di accordo e di posizione dei costituenti, impaccio a gestire la concordanza dei tempi e laspettualità: evidentemente le distanze tra i due sistemi linguistici giocano un ruolo fondamentale e non possono prescindere da considerazioni morfologiche, pragmatiche e testuali. Quest'area dell'interlingua mostra infatti una tendenza all'errore che aumenta insieme allo sviluppo dei mezzi di coerenza e coesione del testo, alla sua dimensione logica e referenziale, e per questo si ritiene che, in prospettiva didattica, possa essere sostenuta da un approccio pragmatico-testuale, anche in chiave contrastiva.

Le osservazioni sul lessico emerse confermano lo studio di Bozzone Costa (2002). Questo potrebbe indurre a concludere che l'analisi dell'errore lessicale su base contrastiva - e a partire da un canale comunicativo come quello scritto - non porti a risultati particolarmente rilevanti; tuttavia, proprio le ambiguità legate a strategie di calco e prestito da L1 potrebbero suggerire regolarità e generalizzazioni più significative, se elicitate da dati ricavati da un numero di informanti più consistente. Non ultimo, le devianze di stile/registro prodotte nel livello intermedio iniziale portano a considerare la competenza scritta legata alla stesura dei diversi tipi di testo come un aspetto dell'apprendimento in L2 da non trascurare (cfr. anche Berman 1994).

Nellortografia si rilevano molti errori soprattutto nella varietà iniziale e riguardano la scrittura delle consonanti geminate, lelisione e la scelta delle varianti degli articoli determinativi e indeterminativi; la frequenza degli errori per quel che riguarda la scrittura delle consonanti doppie non si esaurisce nel livello intermedio e questo porta a considerare la competenza ortografica come un aspetto della lingua che occorre curare anche nello stadio più avanzato attraverso attività didattiche mirate a consolidare la conoscenza degli aspetti fonologici (e di conseguenza, ortografici) della L2.

36 Come detto, l'interferenza da inglese L2 - ormai parte del background linguistico di tutti gli apprendenti, soprattutto europei - è presente insieme a quella della L1, tanto da indurre a pensare che si tratti di una costante che potrebbe essere acquisita all'interno delle sequenze acquisizionali valida per tutte le L1. 
Gli errori di morfologia, superato lo stadio iniziale, risultano essere in maggior proporzione nella varietà avanzata, e sembrano essere determinati da transfer negativo o fossilizzazione che portano ad aggiungere al verbo - in particolar modo copulativo e intransitivo - le forme del pronome personale atono di terza persona (lui si torna invece di lui torna). Sempre il transfer sembra guidare la scelta di genere del nome anche a livelli avanzati. Il dato evidenziato in 3.4 si allinea infatti ai risultati emersi da alcuni studi degli ultimi anni (come Bestgen \& al. 2012; Ellis 2008) secondo i quali il transfer agisce in maniera preponderante anche nei livelli più avanzati dell'apprendimento. A questo proposito, potrebbe essere interessante analizzare il fenomeno dell'aggiunta del pronome personale atono $s i$-riflessivo a verbi copulativi e intransitivi per ricercarne le cause: i dati farebbero pensare a un fenomeno di transfer legato all'utilizzo del pronome di terza pers. singolare e plurale (molto vicino alle forme 'se' - pronome atono invariato ceco - e 'sa' - pronome atono invariato slovacco) oppure a fossilizzazione.

Gli errori di punteggiatura prevalgono nei testi di livello intermedio iniziale e risultano comunque preponderanti anche nella fase di apprendimento successiva, evidenziando come l'applicazione delle funzioni interpuntorie sia soggetta a transfer da L1 e vada di pari passo con lo sviluppo della complessità delle proposizioni. La punteggiatura si è confermata quindi come un aspetto critico della testualità che andrebbe curato al pari delle altre aree della lingua a partire già dai livelli più bassi del percorso acquisizionale: questo anche per evitare fenomeni di fossilizzazione nei livelli più avanzati per quel che riguarda l'espressione logica e sintattica dei segni interpuntori e per guidare, nei livelli più avanzati, all'uso della funzione informativa.

Per concludere, si auspica che le osservazioni qui avanzate possano essere ulteriormente confermate $\mathrm{o}$, perché no?, che possano essere utili a generare discussione e sviluppare così la ricerca: le aree di criticità rilevate possono ancora dire molto sull'acquisizione, sulle difficoltà e sulle competenze testuali di apprendenti con L1 ceca e slovacca.

\section{Riferimenti bibliografici}

Andorno, C.; \& Rastelli, S. (2009). Un’annotazione orientata alla ricerca acquisizionale. In C. Andorno \& S. Rastelli (Eds.), Corpora di italiano L2 (pp. 49-70). Perugia: Guerra Edizioni.

Barni, M.; Bandini, A.; Sprugnoli, L.; Lucarelli, S.; Scaglioso, A. M.; Strambi, B.; Fusi, C.; \& Arruffoli, A. (2009). Linee guida CILS. Perugia: Guerra Edizioni.

Bachniková, A.; Benešová, H.; \& Ehrenbergerová, L. (2008). Italština. Praha: Leda.

Berman, R. (1994). Learners' transfer of writing skills between languages. TESL Canada Journal, 12, 1, 29-46. Bestgen, Y.; Granger, S.; \& Thewissen, J. (2012). Error Patterns and Automatic L1 Identification. In S. A. Crossley \& S. Jarvis (Eds.), Approaching Language Transfer Through Text Classification: Explorations in the Detection-based Approach (pp. 95-115). Bristol: Multilingual Matters.

Bitonti A.; \& De Tommaso V. (in stampa). Dinamiche di interferenza in contesto di apprendimento. Il tempo e l'aspetto verbale in italiano e in ceco. In M. Borreguero Zuloaga et al. (Eds.). Acquisizione e didattica dell'italiano, Atti del XIV Congresso SILFI (Madrid, 04.-06.04.2016).

Bozzone Costa, R. (2002). Rassegna degli errori lessicali in testi scritti da apprendenti elementari, intermedi ed avanzati di italiano L2 (ed implicazioni didattiche). Linguistica e Filologia, 14, 37-67. 
Calleri, D. (2008). Le preposizioni italiane: una categoria “debole”. In G. Bernini, L. Spreafico \& A. Valentini (Eds), Competenze lessicali e discorsive nellacquisizione di lingue seconde, Atti del Convegno-Seminario Bergamo, 8-10 giugno 2006 (pp. 37-52). Perugia: Guerra Edizioni.

Callies, M.; \& Götz, S. (2015). Learner corpora in language testing and assessment. Prospects and challenges. In M. Callies, \& S. Götz (Eds.), Learner Corpora in Language Testing and Assessment (pp. 1-9). Amsterdam-Philadelphia: John Benjamins Publishing Company.

Cattana, A.; Nesci, M. T. (2004). Analizzare e correggere gli errori. Perugia: Guerra Edizioni.

Chini, M. (2005). Che cosè la linguistica acquisizionale. Roma: Carocci.

Chini, M.; \& Bosisio, C. (2014). Fondamenti di glottodidattica. Apprendere e insegnare le lingue oggi. Roma: Carocci.

Corder, P. S. (1981). Error analysis and interlanguage. Oxford: OUP.

Dagneaux, E.; Denness, S.; \& Granger, S. (1998). Computer-aided error analysis. System: An International Journal of Educational Technology and Applied Linguistics, 26, 163-174. https://doi.org/10.1016/S0346251X(98)00001-3.

Ellis, R. (2008). The Study of Second Language Acquisition. Oxford: OUP.

Ferrari, A. (2014). Linguistica del testo. Principi, fenomeni, strutture. Roma: Carocci.

Ferrari, A.; \& Zampese, L. (2016). Grammatica: parole, frasi, testi dell'italiano. Roma: Carocci.

Giacalone Ramat, A. (1993). Italiano di stranieri. In A. A. Sobrero (Ed.), Introduzione all'italiano contemporaneo. La variazione e gli usi (pp. 341-409). Roma-Bari: Laterza.

Giacalone Ramat, A. (Ed.). (2003). Verso l'italiano. Percorsi e strategie di acquisizione. Roma: Carocci.

Hartmannová, V.; \& Hartmannová D. (1994). Pravidla českého pravopisu. Olomouc: Nakladatelství FIN.

Havránek, B.; \& Jedlička, A. (1981). Česká mluvnice. Praga: Státní pedagogické nakladatelství.

Hůrková-Novotná, J. (1995). Česká výslovnostní norma. Praha: Scientia.

Klimová, K. (2011). Questioni di aspetto verbale. Un confronto tra italiano e slovacco. Roma: Aracne Editrice.

Klímová, E. (2011). On semantic scales in Italian in comparison with English and Czech. Slavica viterbiensia, III, 79-93.

. (2013). Principi dellordine delle parole in tre lingue tipologicamente diverse. In M. Rückl, E. Santoro \& I. Vedder (Eds.), Contesti di apprendimento L2. Tra teoria e pratica didattica (pp. 43-58). Firenze: Franco Cesati Editore.

Pallotti, G. (1998). La seconda lingua. Milano: Bompiani.

- (2005). Interlingua e analisi degli errori. MIUR-ANSAS: Piattaforma di formazione Poseidon.

Quadro comune europeo di riferimento per le lingue: apprendimento, insegnamento, valutazione. (2002). Milano: RCS Scuola.

Rodríguez García, C. (2014). Análisis de errores léxicos en la producción escrita. Una valoración comunicativa. In M. Aurová, J. Pešková, M. J. Santiago Gutiérrez \& J. Prokop (Eds.), Al pie de la(s) letra(s), Sborník př́spěvků ze Setkání hispanistů na JU v Českých Budějovicích 2014. České Budějovice: Filozofická fakulta Jihočeské univerzity v Českých Budějovicích.

Rodríguez García, C. (in stampa). Tipología de errores en la expresión escrita. In 4th International Symposium Language for International Communication: Linking Interdisciplinary Perspectives on Cultural, Professional and Scientific Capacity Building. Riga, University of Latvia.

Sokolová, M.; Musilová, K.; \& Slančová, D. (2005). Slovenčina a čeština: synchrónne porovnanie s cvičeniami. Bratislava: Univerzita Komenského. 
Trovesi, A. (2013). Divergenze e convergenze tra ceco e slovacco. Un profilo storico e linguistico. In A. Di Gregorio (ed.), Il ventennale dello scioglimento pacifico della Federazione Ceco-Slovacca: profili storico-politici, costituzionali, internazionali (pp. 79-106). Milano: Maggioli.

Uhlířová, L. (2017). Slovosled nominální skupiny. In P. Karlík, M. Nekula \& J. Pleskalová (Eds.), CzechEncy - Nový encyklopedický slovník češtiny. https://www.czechency.org/slovnik/SLOVOSLED NOMINÁLNÍ SKUPINY.

Young, R. (1996). Form-Function Relations in Articles in English Interlanguage. In R. Bayley \& D.R. Preston (Eds.), Second language acquisition and linguistic variation (pp. 135-175). Amsterdam-Philadelphia: John Benjamins. 